Sandra Maria do Valle Leone de Oliveira'

Michael Robin Honer ${ }^{2}$

Anamaria Mello Miranda Paniago ${ }^{3}$

Eliana Setti Aguiar ${ }^{4}$

Rivaldo Venâncio da Cunha ${ }^{5}$

\section{Efeito booster na prova tuberculínica em um hospital universitário de Mato Grosso do Sul}

\author{
Booster effect on tuberculin skin tests at a university hospital in \\ Mato Grosso do Sul
}

\author{
${ }^{1}$ Mestre em Saúde Coletiva, enfermeira \\ do Hospital Dia Prof. Esterina Corsini \\ - Universidade Federal do Mato Grosso \\ do Sul. \\ 2Professor Doutor do Departamento de \\ Saúde Coletiva da Universidade Federal \\ do Mato Grosso do Sul. \\ ${ }^{3}$ Professora Doutora Adjunta do \\ Departamento de Clínica Médica da \\ Universidade Federal do Mato Grosso \\ do Sul. \\ ${ }^{4}$ Professora Mestre Adjunta do Depar- \\ tamento de Clínica Médica da Universi- \\ dade Federal do Mato Grosso do Sul. \\ ${ }^{5}$ Professor Doutor Associado do \\ Departamento de Clínica Médica da \\ Universidade Federal do Mato Grosso \\ do Sul. \\ Contato: \\ Sandra Maria do Valle Leone de Oliveira \\ Rua Marques de Pombal, 1889, apto. 3, \\ bloco A - Condomínio Novo Portugal \\ Bairro Tiradentes. Campo Grande \\ - Mato Grosso do Sul \\ E-mail: \\ sandrinhaleone@gmail.com
}

Recebido: 30/07/2007

Revisado: 28/01/2008

Aprovado: 26/02/2008

\section{Resumo}

A triagem tuberculínica tem por objetivo realizar o rastreio da infecção tuberculosa. Uma retestagem em uma a três semanas tem sido recomendada quando a prova tuberculínica for $<10 \mathrm{~mm}$ na $1^{\mathrm{a}}$ dose para averiguar a ocorrência do efeito booster. Quando a prova tuberculínica for $<10 \mathrm{~mm}$ na $1^{\mathrm{a}}$ dose e, uma a três semanas depois, na $2^{\mathrm{a}}$ dose, ocorrer uma enduração $\geq 10 \mathrm{~mm}$ com aumento de pelo menos $6 \mathrm{~mm}$ em relação à primeira dose, tem-se, então, o efeito booster. Este estudo teve como sujeitos os profissionais de saúde de um hospital universitário e foi realizado na cidade de Campo Grande-MS com o objetivo de estimar a taxa de efeito booster. Dentre os 194 participantes, 65 (33,5\%) foram reatores fortes ao PPD na primeira testagem e 129 foram reatores fracos ou não-reatores. Estes foram submetidos a uma segunda testagem, na qual, em 10 $(7,8 \%)$ observou-se o efeito booster. A prevalência da infecção tuberculosa, avaliada em dois tempos, foi de 38,7\%. Conclui-se que identificar o efeito booster na triagem tuberculínica é importante sob pena de obter-se taxas subestimadas de infecção tuberculosa. Útil também no acompanhamento da infecção recente e na avaliação segura da viragem tuberculínica, reduzindo a probabilidade de falsos negativos, que de outra forma seriam erroneamente interpretados como profissionais recém-infectados.

Palavras-chaves: prova tuberculínica cutânea, prevalência, profissionais de saúde, fenômeno booster.

\begin{abstract}
Tuberculin screening aims at tracking tuberculosis infection. A one-to-threeweek retest has been recommended when the tuberculin test is $<10 \mathrm{~mm}$ in the first dose to check booster effect occurrence. The booster phenomenon occurs when the tuberculin screening is $<10 \mathrm{~mm}$ in the first dose and after the second dose, in three weeks time, there is a $>10 \mathrm{~mm}$ induration with an increase compared to the first dose of at least $6 \mathrm{~mm}$. This study, aimed at estimating booster effect rate, involved health professionals from a University hospital in Campo Grande, a town in the Brazilian state of Mato Grosso do Sul. Among the 194 participants, 65 (33,5\%) were strong PPD reactors in the first test and 129 were weak or non-reactors. They went through a second test which revelled 10 were reactors. Hence, the booster effect rate was 7,8\%. In the two-step tuberculin test reactor tuberculosis infection prevalence was $38.7 \%$. The authors concluded that identifying booster effect in tuberculin screening is important to avoid under estimated rates of tuberculosis infection. It is also important to follow up recently acquired infections and evaluate the results of tuberculin screening accurately to reduce the probability of false negative results, which would otherwise be misinterpreted as refering to recently infected health professionals.
\end{abstract}

Keywords: tuberculin skin test, prevalence, health care professionals, booster phenomenon. 


\section{Introdução}

A triagem tuberculínica é uma medida que tem por objetivo realizar o rastreio de profissionais com infecção tuberculosa. Também é considerada uma estratégia de detecção e prevenção de novos casos na transmissão intra-hospitalar, constituindo-se em um importante instrumento complementar no conjunto de ações de vigilância e diagnóstico da tuberculose (BEDRIKOW et al., 1977; SOCIEDADE BRASILEIRA DE PNEUMOLOGIA E TISIOLOGIA, 2004; CENTER FOR DISEASE CONTROL AND PREVENTION, 2005).

Na avaliação da infecção tuberculosa pela prova tuberculínica, o profissional de saúde é considerado infectado pelo Mycobacterium tuberculosis quando apresentar enduração $\geq 10 \mathrm{~mm}$, avaliada 72-96 horas após a injeção intradérmica de 2 UI de PPD RT 23. Um resultado entre 5-9 $\mathrm{mm}$ deve ser interpretado como um teste fracamente positivo, podendo ser resultado da vacinação com BCG ou da infecção por outras micobactérias não-tuberculosas.

Um teste subseqüente positivo, após duas testagens negativas, avaliado após 12 meses, será considerado viragem tuberculínica e o tratamento da infecção tuberculosa poderá ser recomendado (SOCIEDADE BRASILEIRA DE PNEUMOLOGIA E TISIOLOGIA, 2004).

Quando a prova tuberculínica for $<10 \mathrm{~mm}$ na $1^{\mathrm{a}}$ dose e, 1 a 3 semanas depois, na $2^{\text {a }}$ dose, ocorrer uma enduração $\geq 10 \mathrm{~mm}$ com aumento de pelo menos 6 mm em relação à primeira dose, tem-se, então, o efeito booster. Isso ocorre, possivelmente, por causa da diminuição da resposta de hipersensibilidade tardia após um longo período de instalação da infecção tuberculosa (CENTRO DE VIGILÂNCIA EPIDEMIOLÓGICA, 2001).

O aumento da enduração comparada à primeira testagem pode causar uma falsa impressão de conversão ao teste. O que deve de fato ser entendido é que a reatividade às micobactérias dormentes ou latentes foi restaurada pelo estímulo antigênico gerado pela prova inicial (MELLO et al., 2003).

Este fenômeno pode levar também ao aumento do tamanho da reação na avaliação subseqüente e é mais habitual em pessoas acima de 60 anos, podendo falsamente identificar uma pessoa como recém-infectada (KRAUT et al., 2004; CHOUDHARY et al., 2006).

A realização da prova tuberculínica em duas fases é indicada quando se prevê uma realização seqüencial para acompanhamento de profissionais de saúde e dos pacientes infectados pelo vírus HIV ou imunocomprometidos, tais como doentes renais que realizam hemodiálise e indivíduos residentes em asilos (CENGIZ; SEKER, 2006).

O objetivo do presente estudo foi (re)conhecer a taxa de efeito booster em uma população de profissio- nais de saúde de um hospital universitário de Mato Grosso do Sul.

\section{Material e métodos}

A pesquisa foi realizada no município de Campo Grande-MS, em um hospital-escola que desenvolve atividades de ensino e pesquisa, o qual é uma entidade autárquica federal, mantida por recursos dos Ministérios da Educação e da Saúde.

O Hospital Universitário (HU) da Universidade Federal de Mato Grosso do Sul (UFMS) desenvolve atenção terciária e é campo de ensino para graduandos de Enfermagem, Odontologia, Medicina e Farmácia, cursos de Ensino Médio, como Técnico de Enfermagem, Técnico em Laboratório e Técnico em Radiologia.

A população deste estudo foi constituída pelos trabalhadores do HU da UFMS que tinham contato, no ambiente intra-hospitalar, direta ou indiretamente, com pacientes no processo de internação ou com suas secreções em ambiente de laboratório. O período de estudo foi de 01 de abril a 30 de setembro de 2004 .

No Brasil, o Ministério da Saúde estima que cerca de $30 \%$ da população seja infectada pelo Mycobacterium tuberculosis, freqüência que foi utilizada para fins de cálculo da amostra no presente estudo.

A amostra de 194 servidores foi calculada no Programa EpiInfo 2003, 3.3.2, utilizando-se a técnica de amostragem probabilística estratificada com a inserção de mais $20 \%$ para as correções de perdas.

O critério de inclusão foi: consentir a participação, assinando o Termo de Consentimento Livre e Esclarecido após receber informação verbal sobre os objetivos e a metodologia do trabalho. Ter história prévia de tuberculose (doença), uso prévio de tuberculostático, terapia imunossupressora em vigência e não consentir a participação no estudo, na primeira ou na segunda fase, foram considerados critérios de exclusão.

Utilizou-se o formulário, previamente validado, do Programa de Controle de Tuberculose Hospitalar da Universidade Federal do Rio de Janeiro (MELLO et al., 2003).

Para realizar a prova tuberculínica, foi usada a técnica de Mantoux, injetando-se 0,1 ml de PPD RT 23 (purified protein derivate) intraderme na face anterior do antebraço esquerdo de cada profissional, formando uma pápula de bordas bem delimitadas.

A avaliação da reatividade foi instituída a partir da região de enduração, medida pelo método palpatório, 72 a 96 horas após a injeção. Nos profissionais não-reatores na primeira prova, ou seja, quando a enduração do PPD foi igual ou inferior a $9 \mathrm{~mm}$, pesquisou-se o efeito do tipo booster. 
Considerou-se resposta do tipo booster ou fenômeno booster quando a prova tuberculínica foi $<10 \mathrm{~mm}$ na $1^{\mathrm{a}}$ dose e, na $2^{\mathrm{a}}$ dose, feita de 1 a 3 semanas depois, apresentou enduração $\geq 10 \mathrm{~mm}$ e aumento de pelo menos $6 \mathrm{~mm}$ em relação à primeira dose (SEPKOWITZ et al., 1997; SOCIEDADE BRASILEIRA DE PNEUMOLOGIA E TISIOLOGIA, 2004). Todos os indivíduos que apresentaram a prova tuberculínica reator forte foram encaminhados para avaliação médica especializada.

O presente estudo foi aprovado no comitê de ética em pesquisas envolvendo seres humanos da UFMS.

Os resultados foram analisados utilizando-se o programa Epilnfo Windows 3.3.2, 2004. A associação das variáveis categóricas foi avaliada por meio do teste do qui-quadrado e pelo teste exato de Fisher quando $\mathrm{n}<5$.

\section{Resultados}

Dentre os 194 participantes, 33,5\% (65/194) foram fortes-reatores ao PPD na primeira testagem. Aqueles fracos ou não-reatores (129/194) foram submetidos a uma segunda testagem, que resultou em 10 fortesreatores, obtendo-se, desta forma, uma taxa de efeito booster de 7,8\% (10/129) (Fluxograma 1).

A prevalência da infecção tuberculosa, em dois tempos, foi de $38,7 \%(75 / 194)$ de reatores.

\section{Discussão}

Thompson et al., na década de 1970, reforçaram a necessidade de utilização da testagem repetida para distinguir as reações decorrentes do fenômeno booster daquelas originadas por uma infecção recente (THOMPSON, 1979; MELLO, 2003).
Neste estudo foi observada uma taxa de efeito booster de 7,8\%.

Taxas variando de $0 \%$ a $9,7 \%$ foram demonstradas em estudos conduzidos pelo Centers for Disease Control and Prevention em dez hospitais americanos (SEPKOWITZ et al., 1997). No México, Zeichner et al. (2000) encontraram, em um hospital universitário, uma taxa de efeito booster de 10\% (49/483). No Brasil, estudos realizados no Rio de Janeiro demonstraram taxa de efeito booster variando de 5,8\% a $8,4 \%$ (MUZZY DE SOUZA et al., 1998; SILVA et al., 2001; SOARES; KRITSKI, 2004).

A prevalência global de fortes-reatores foi de $38,7 \%$. Por outro lado, 8,8\% (17/194) foram considerados fracos-reatores, resultados entre 5-9 mm. Estes resultados fracos-reatores podem ser atribuídos à infecção por outras micobactérias não-tuberculosas, à vacinação com BCG intradérmica ou a uma baixa resposta ao estimulo antigênico pelo Mycobacterium tuberculosis.

Na prevalência global, devem ser considerados os aspectos ambientais, o número de pacientes bacilíferos atendidos no serviço, o tempo de exposição do paciente aos profissionais, que são fatores que incrementam o risco de transmissão no hospital.

No presente estudo, não houve associação estatisticamente significativa dos profissionais com infecção tuberculosa com variáveis sexo, idade (Tabela 1), vacinação de BCG e presença de cicatriz vacinal.

Choudhary et al. (2006) observam que a testagem em duas etapas não incrementa o custo no acompanhamento anual dos profissionais, considerando que esta estratégia elimina os falsos-positivos em avaliações anuais, os quais, inadvertidamente, poderiam ser submetidos a quimioprofilaxia sem necessidade (MELO; AFIUNE, 1985; KRITSKI et al., 1993).

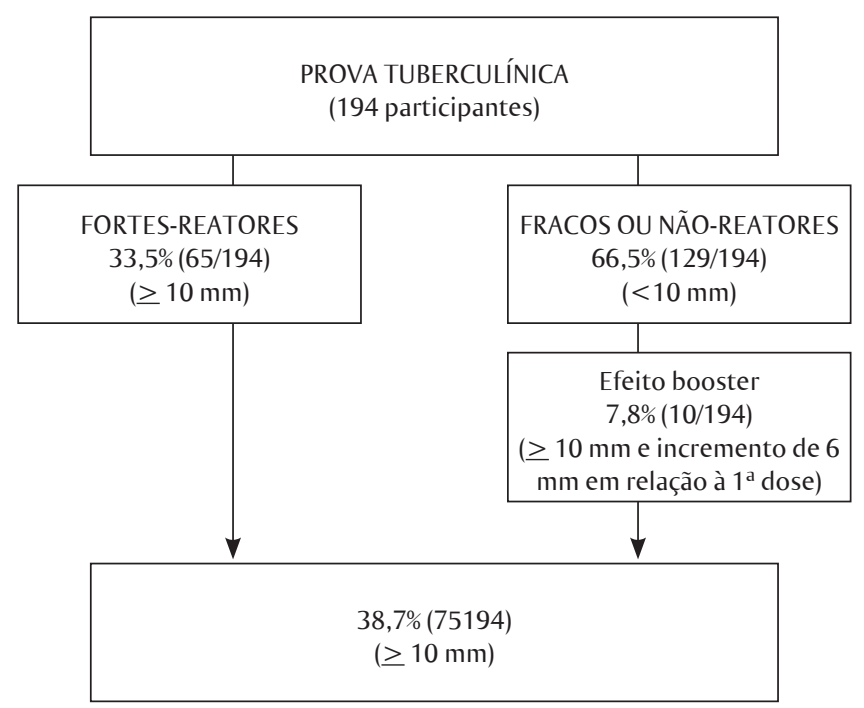

Figura 1 Resultados da triagem tuberculínica realizada em trabalhadores do Hospital Universitario, UFMS, 2004 
Tabela 1 Sexo, idade e efeito booster em profissionais de saúde do Núcleo do Hospital Universitário Maria Aparecida Pedrossian / UFMS, 2004

\begin{tabular}{|c|c|c|c|c|}
\hline \multirow[b]{2}{*}{ Variáveis } & \multicolumn{2}{|c|}{ Efeito Booster } & \multirow{2}{*}{$\begin{array}{c}\text { Total } \\
n(\%)\end{array}$} & \multirow[b]{2}{*}{$p$} \\
\hline & $\begin{array}{c}\text { Booster } \\
n(\%)\end{array}$ & $\begin{array}{c}\text { Não booster } \\
n(\%)\end{array}$ & & \\
\hline \multicolumn{5}{|l|}{ Sexo } \\
\hline Masculino & $3(4,8)$ & $60(95,2)$ & $63(100,0)$ & 0,58 \\
\hline Feminino & $7(5,3)$ & $124(94,7)$ & $131(100,0)$ & \\
\hline \multicolumn{5}{|l|}{ Idade } \\
\hline$<36$ anos & $4(3,9)$ & $98(96,1)$ & $102(100,0)$ & 0,62 \\
\hline$\geq 36$ anos & $6(6,5)$ & $86(93,5)$ & $92(100,0)$ & \\
\hline
\end{tabular}

Nota: $\mathrm{p}<0,05$. Teste qui-quadrado corrigido Yates. Foi usado o Exato de Fisher Bicaudal para o valor de p.

\section{Conclusão}

Para avaliar a magnitude da infecção tuberculosa entre profissionais de saúde, é imprescindível que os programas de vigilância e controle conheçam a realidade epidemiológica.

Concluímos que é recomendável a repetição da prova tuberculina em dois tempos como parte das ações do programa de controle de tuberculose e do programa de saúde ocupacional em hospitais. A identificação do fenômeno booster é uma medida fundamental no (re)conhecimento da infecção tuberculosa em profissionais recém-admitidos, reduzindo a probabilidade de falsos-negativos, que, de outra forma, seriam erroneamente interpretados como profissionais recém-infectados.

\section{Referências}

BEDRIKOW, B. et al. Freqüência da tuberculose entre funcionários de uma instituição de assistência médica e os resultados parciais de um programa de controle. Rev. bras. Saúde ocup., São Paulo, v. 5, n. 20, p. 30-33, out./dez. 1977.

CENTRO DE VIGILÂNCIA EPIDEMIOLÓGICA. Manual de Atualização: treinamento nas técnicas de aplicação e leitura do teste tuberculínico. São Paulo: CVE, 2001. 28 p.

\section{CENTER FOR DISEASE CONTROL AND}

PREVENTION. Guidelines for preventing the transmission of mycobacterium tuberculosis in health-care settings, 2005. Morbity and Mortality Weekly Report. v. 54, n. RR-17, Dec., 2005. Disponível em: http://www.cdc.gov/mmwr/pdf/rr/rr5417.pdf. Acesso em: 30 set. 2007.

CENGIZ, K.; SEKER, A. Boosted tuberculin skin testing in hemodialysis patients. Am. J. Infect. Control., United States, v. 34, n. 6, p. 383-387, Aug. 2006.

CHOUDHARY , M. et al. A university hospital 10year experience with tuberculin testing: value of the 2-step tuberculin skin test. Am. J. Infect. Control., United States, v. 34, n. 6, p. 358-361, 2006.
KRITSKI, A. L. et al. Tuberculose entre profissionais de saúde. Risco ocupacional? J. Pneumologia, v. 19, p. 113-121, 1993.

KRAUT, A. et al. Predictors of positive tuberculin skin test (TST) results after 2-step TST among health care workers in Manitoba, Canada. Clin. Infect. Dis., United States, v. 39, n. 11, p. 113-118. Dec. 2004.

MELO, F. A. F.; AFIUNE, J. B. Tuberculose, uma doença ocupacional: infecção, adoecimento e proteção dos profissionais de saúde em serviços de atenção a tuberculose. B. Pneumologia Sanit., Rio de Janeiro, v. 3, n. 1, p. 56-68, jan./jun. 1985.

MELLO, D. A. et al. O fenômeno booster na avaliação da prova tuberculínica cutânea. Pulmão, Rio de Janeiro, v. 12, n. 3, p. 128-130, 2003.

MUZZY DE SOUZA, G. R. et al. Tuberculin conversion among health care workers in a general hospital of Rio de Janeiro, Brazil. Am. J. Respir. Crit. Care Med., v. 2 (suppl.), p. 387, 1998.

SEPKOWITZ, K. A. et al. Benefit of two-step PPD testing of new employees at a New York City hospital. Am. J. Infectio Control., v. 25, n. 3, p. 283286, 1997. 
SILVA, V. J. R.; SANTOS, F. M. S. A.; KRITSKI, A. L. Prevalência de infecção Mycobacterium tuberculosis entre alunos da Faculdade de Medicina da Universidade Federal do Rio de Janeiro. J. Pneumologia, São Paulo, v. 27, n. 2, p. 77-82, 2001.

SOARES, L. M. F.; KRITSKI, A. Prevalência da prova tuberculínica positiva entre alunos da Faculdade de Medicina de Campos (RJ). J. bras. Pneumologia, São Paulo, v. 30, n. 4, p. 440-447, 2004.

SOCIEDADE BRASILEIRA DE PNEUMOLOGIA E TISIOLOGIA. II Concenso brasileiro de tuberculose
- diretrizes brasileiras para tuberculose. J. bras. Pneumologia, São Paulo, v. 30, Suplemento 1, jun. 2004.

THOMPSON, N. J. et al. The booster phenomenon in serial tuberculin testing. Am. Rev. Resp. Dis., United States, v. 119, n. 4, p. 587-597, 1979.

ZEICHNER, L. O. F. et al. Tuberculosis en trabajadores de la salud: importancia de los programas de vigilancia y control. Salud Pub. Mex., v. 42, n. 1, p. 48-52. enero/feb. 2000. 